\title{
Human Adult Cortical Reorganization and Consequent Visual Distortion
}

\author{
Daniel D. Dilks, ${ }^{1,3}$ John T. Serences, ${ }^{2,4}$ Benjamin J. Rosenau, ${ }^{2}$ Steven Yantis, ${ }^{2}$ and Michael McCloskey ${ }^{1}$ \\ Departments of ${ }^{1}$ Cognitive Science and ${ }^{2}$ Psychological and Brain Sciences, Johns Hopkins University, Baltimore, Maryland 21218, ${ }^{3}$ McGovern Institute for \\ Brain Research, Massachusetts Institute of Technology, Cambridge, Massachusetts 02139, and ${ }^{4}$ Department of Cognitive Sciences, University of California, \\ Irvine, California 92697
}

Neural and behavioral evidence for cortical reorganization in the adult somatosensory system after loss of sensory input (e.g., amputation) has been well documented. In contrast, evidence for reorganization in the adult visual system is far less clear: neural evidence is the subject of controversy, behavioral evidence is sparse, and studies combining neural and behavioral evidence have not previously been reported. Here, we report converging behavioral and neuroimaging evidence from a stroke patient (B.L.) in support of cortical reorganization in the adult human visual system. B.L.'s stroke spared the primary visual cortex (V1), but destroyed fibers that normally provide input to V1 from the upper left visual field (LVF). As a consequence, B.L. is blind in the upper LVF, and exhibits distorted perception in the lower LVF: stimuli appear vertically elongated, toward and into the blind upper LVF. For example, a square presented in the lower LVF is perceived as a rectangle extending upward. We hypothesized that the perceptual distortion was a consequence of cortical reorganization in V1. Extensive behavioral testing supported our hypothesis, and functional magnetic resonance imaging (fMRI) confirmed V1 reorganization. Together, the behavioral and fMRI data show that loss of input to V1 after a stroke leads to cortical reorganization in the adult human visual system, and provide the first evidence that reorganization of the adult visual system affects visual perception. These findings contribute to our understanding of the human adult brain's capacity to change and has implications for topics ranging from learning to recovery from brain damage.

Key words: cortical reorganization; cortical plasticity; primary visual cortex; perceptual distortion; adult human visual system; visual perception

\section{Introduction}

Some of the first evidence for adult cortical reorganization came from animal and human studies in which a region of primary somatosensory cortex (S1) was deafferented (deprived of input) by means of amputation (Kalaska and Pomeranz, 1979; Merzenich et al., 1983; Merzenich et al., 1984; Calford and Tweedale, 1988; Pons et al., 1991; Elbert et al., 1994; Yang et al., 1994; Flor et al., 1995). Neurons in the deafferented region began responding to stimuli that normally activated adjacent S1 cortex. Other researchers have used perceptual changes (i.e., "referred" sensations) as a marker for reorganization (Ramachandran et al., 1992; Halligan et al., 1993; Aglioti et al., 1994), and still later work provided converging behavioral and functional magnetic resonance imaging ( $\mathrm{fMRI}$ ) evidence for reorganization in the adult somatosensory system (Borsook et al., 1998).

Received Jan. 13, 2006; revised July 17, 2007; accepted July 19, 2007.

This work was supported by a National Science Foundation Integrative Graduate Education and Research Traineeship (D.D.D.), a National Science Foundation Graduate Research Fellowship (J.T.S.), and National Institutes of Health Grant R01-DA13165 (S.Y.). We express our sincere thanks to B.L. and his family. We also thank Argye Hillis for bringing B.L. to our attention, James Hoffman for the use of his laboratory, and Neha Bhatia, Cynthia Chen, Joshua Connor, Eric Gou, Emma Gregory, Jiyeon Jennee Kim, Allison Leung, and Alfred Yu for their help in data collection, coding, and analysis.

Correspondence should be addressed to Daniel D. Dilks, McGovern Institute for Brain Research, Massachusetts Institute of Technology, 46-4141G, Cambridge, MA 02144. E-mail: dilks@mit.edu.

DOI:10.1523/JNEUROSCI.2650-07.2007

Copyright $\odot 2007$ Society for Neuroscience $\quad$ 0270-6474/07/279585-10\$15.00/0
In contrast, solid evidence for reorganization in the adult visual system has not been reported. Initial neural evidence came from adult animal studies in which a region of the primary visual cortex (V1) was deafferented by means of retinal lesions (Kaas et al., 1990; Heinen and Skavenski, 1991; Gilbert and Wiesel, 1992; Chino et al., 1992; Darian-Smith and Gilbert, 1995; Schmid et al., 1996; Calford et al., 2000). Like deafferented neurons in the S1, deafferented V1 neurons began responding to stimuli that normally activated the adjacent V1 cortex. However, some studies have failed to find V1 reorganization in the adult animal (Horton and Hocking, 1998; Smirnakis et al., 2005a). Conflicting results have also emerged from two fMRI studies examining reorganization after deafferentation of human adult visual cortex in patients with macular degeneration (Sunness et al., 2004; Baker et al., 2005).

Behavioral evidence for cortical reorganization in the adult visual system is extremely sparse. With adult human observers Kapadia et al. (1994) found that the position of a line segment presented just below an artificially induced scotoma was shifted toward the scotoma.

We report a study of a stroke patient, B.L., that provides converging behavioral and neural evidence for cortical reorganization in the adult human visual system. V1 is intact in B.L., but his stroke destroyed the fibers that provide input to the $\mathrm{V} 1$ region representing the upper left visual field (LVF). As a consequence, 
B.L. is blind in his upper LVF. Preliminary testing revealed distorted perception in the lower LVF: stimuli appeared vertically elongated into the blind upper LVF. For example, a circle presented in the lower LVF was perceived as cigar shape extending upward. We hypothesized that the perceptual distortion was a consequence of cortical reorganization: if the deafferented V1 region (representing the upper LVF) had begun responding to stimuli that normally activated adjacent cortex (representing the lower LVF), then stimuli presented to the lower LVF might appear perceptually elongated toward and into the blind upper LVF. Extensive behavioral testing supported this hypothesis, and fMRI data confirmed that the deprived V1 region (representing the upper LVF) became responsive to stimuli presented to the lower LVF. Together, our results constitute strong evidence of cortical reorganization in the adult human visual system, and the first evidence establishing a link between cortical reorganization and visual perception.

\section{Materials and Methods Case history}

B.L. is a 51-year-old man who suffered a stroke in December, 2001, 6 months before the beginning of this study. Structural MRI revealed damage to right-hemisphere inferior optic radiations, the fibers that carry upper LVF information from the lateral geniculate nucleus of the thalamus to V1, but no involvement of optic nerve, optic tract, lateral geniculate nucleus (LGN), or V1 (supplemental Fig. 1, available at www.jneurosci.org as supplemental material). Other damage included the right deep temporooccipital lobe involving the fusiform gyrus and thalamus (not including the LGN), and the right corpus callosum affecting the splenium. Visual acuity was normal (20/30 right eye, 20/30 left eye, 20/30 both eyes). Pupils reacted normally, extraocular movements were full, and there was no nystagmus. Dilated fundus examination revealed clear media with normal disks, maculae, and vessels. Visual field testing in our laboratory revealed a scotoma (blind area) covering most of the upper LVF (except for a spared strip along the vertical meridian) and extending a few degrees into the lower LVF, especially in the periphery (supplemental Fig. 2, available at www.jneurosci.org as supplemental material). The scotoma and perceptual distortions (discussed below) were observed when B.L. was first tested 6 months after the stroke, and remained stable, both qualitatively and quantitatively, over the 4 years of testing.

\section{Behavioral experiments}

Vertical and horizontal judgments. B.L. fixated a central point on a computer monitor, and two white rectangles were presented simultaneously on a black background for $150 \mathrm{~ms}$, one in the lower LVF and one in the lower right visual field (RVF). Rectangles were centered $14^{\circ}$ horizontally from fixation, with the bases aligned $12.6^{\circ}$ vertically below fixation. In each of four 25-trial blocks, all possible pairs of five stimulus rectangles were presented in random order. In the vertical judgment task, the rectangles were $3.2^{\circ}$ wide, but differed in height $\left(3.2^{\circ}, 4.8^{\circ}, 6.4^{\circ}, 8.0^{\circ}\right.$, or $\left.9.6^{\circ}\right)$; for the horizontal judgment task, the height and width dimensions were reversed. B.L. indicated which rectangle was taller (vertical judgment task) or wider (horizontal judgment task) by saying "left," "right," or "same." Four adults with intact vision served as control participants.

Responses were coded by assigning a -1 to each "left" response, $\mathrm{a}+1$ to each "right" response, and a 0 to each "same" response. A mean judgment score for each stimulus size difference (in degrees of visual angle) was computed by averaging the coded responses across trials. A negative mean judgment score indicates a predominance of "left greater" responses, whereas a positive score indicates a predominance of "right greater" responses.

The method of probits was used to identify the point of subjective equality (PSE), that is, the $50 \%$ point on the psychometric function, corresponding to the size difference at which B.L. perceived the two shapes as equal. A positive PSE indicates overestimation of the relevant dimension (i.e., height or width) for stimuli presented in the (affected) lower LVF. For example, a PSE of $3^{\circ}$ in the vertical judgment task would indicate that B.L. perceived the stimuli in a pair to be equal in height when the lower RVF stimulus was actually $3^{\circ}$ taller than the stimulus in the lower LVF. In contrast a negative PSE indicates underestimation of the relevant dimension of lower LVF stimuli. The probit analysis also identified the 95\% confidence interval around the PSE, and the amount of elongation was considered significantly different from zero if the confidence interval did not include zero. Because the method of probits requires a dichotomous dependent variable (e.g., left vs right response), the same responses for each stimulus pair were divided equally between the left and right response categories. This division mimics a twoalternative forced-choice procedure.

Shape judgment. The predicted vertical elongation was also tested using a shape judgment task in which B.L. judged the shape of a single rectangle presented in the lower LVF or lower RVF. Rectangles were centered $14^{\circ}$ horizontally from fixation, with the bases aligned $12.6^{\circ}$ vertically below fixation. In each of three 25 -trial blocks, each rectangle was presented five times in random order. Five rectangles $\left(3.2^{\circ}\right.$ wide, but differing in height: $1.2^{\circ}, 2.2^{\circ}, 3.2^{\circ}, 4.2^{\circ}$, or $5.2^{\circ}$ ) served as stimuli. On each trial, B.L. judged whether the rectangle was "wider than a square" (i.e., width greater than height), "a square," or "taller than a square" (i.e., height greater than width).

Responses were coded by assigning a -1 to each "taller than a square" response, $\mathrm{a}+1$ to each "wider than a square" response, and a 0 to each "square" response. A mean judgment score for each stimulus size difference (in degrees of visual angle) was computed by averaging the coded responses across trials. A negative mean judgment score indicates a predominance of "taller than a square" responses, whereas a positive score indicates a predominance of "wider than a square" responses. The PSE was also computed.

Extent of distortion. A third task examined the extent of distortion as a function of distance from the scotoma. A numerical scale ranging from 1 to 15 was displayed in the (intact) RVF, just to the right of (and touching) the vertical meridian, with 1 and 15 positioned $11.2^{\circ}$ below and above the horizontal meridian, respectively, and 8 on the horizontal meridian. Scale numbers were $1.6^{\circ}$ apart. A square $\left(3.2^{\circ} \times 3.2^{\circ}\right)$ was presented in one of six positions in the lower LVF or lower RVF. The top of the square was $0^{\circ}, 4.8^{\circ}$, or $9.6^{\circ}$ below the horizontal meridian (high, middle, and low vertical placements, respectively), and centered at $2.0^{\circ}$ or $6.0^{\circ}$ from the vertical meridian (near and far horizontal eccentricities, respectively). B.L. reported the number with which the top of the square was aligned.

Tactile shape perception. Stimuli were pairs of wooden rectangles attached to a vertical surface. In the vertical judgment task, the rectangles were $2 \mathrm{~cm}$ wide, but differed in height $(2,3,4,5$, or $6 \mathrm{~cm})$; for the horizontal judgment task, the rectangles were rotated $90^{\circ}$ to vary in the horizontal dimension. All rectangles were $1 \mathrm{~cm}$ thick. B.L. was blindfolded and seated facing the center of the vertical surface. Rectangles were simultaneously presented to the lower left and right of the central point. In each of two 25-trial blocks, all possible pairs of the five rectangles were presented in random order. After simultaneously feeling the blocks (the left block with his left hand and the right block with his right hand), B.L. indicated which rectangle was taller (or wider) by saying "left," "right," or "same." Coding and analyses were the same as in the visual shape perception experiments.

Visual perception of spacing. If cortical reorganization has occurred in $\mathrm{V} 1$, then the distortion should not be selective to perception of shape. Accordingly, we investigated perception of the vertical spacing between two lines. Each stimulus consisted of two white horizontal lines, $3.2^{\circ}$ in length, displayed one above the other. Five stimuli were created by varying the vertical spacing between the lines $\left(1.6^{\circ}, 2.0^{\circ}, 2.4^{\circ}, 2.8^{\circ}\right.$, or $\left.3.2^{\circ}\right)$. In each of four 25-trial blocks, all possible pairs of five stimulus pairs were presented in random order. Pairs of lines were centered $14^{\circ}$ horizontally from fixation, with the bottom line aligned $12.6^{\circ}$ vertically from fixation. On each trial, two pairs of lines were presented, one in the lower LVF and one in the lower RVF. B.L. judged whether the vertical spacing between lines was greater in the left stimulus, greater in the right stimulus, or the same in both. He was instructed to base his judgment on the distance between the lower edge of the top horizontal line and the upper edge of the bottom horizontal line so that any perceived vertical elongation (thickening) of the LVF lines would not lead to overestimation of the 
spacing between lines. Perceived upward extension of the top line should have no effect on the spacing judgment, whereas upward extension of the bottom line should work against finding vertical elongation of space in the LVF. Methods were otherwise the same as in the visual shape perception experiments.

Frame of reference. Because V1 is retinotopically organized, B.L.'s perceptual distortions should reflect a retinocentric reference frame (as opposed to a head-centered, body-centered, or environment-centered frame), if the distortions arise from V1 reorganization. To test this prediction, we conducted two experiments. In the first frame of reference task, B.L. viewed a computer screen with his body upright, but his head tilted $90^{\circ}$ to the right, resting on a horizontal pillow. A stimulus shape (e.g., circle) was presented in the upper left quadrant of the monitor (corresponding to B.L.'s lower LVF), and B.L. was asked to describe the shape. This task dissociates retinocentric and head-centered reference frames from other potential frames (e.g., trunk-centered, screencentered, room-centered). In the second task, B.L. was tested with his head upright but turned to the left or right, so that the entire monitor was on the right or left side of head-centered space, respectively. In both conditions, he kept his eyes fixed on a central point on the monitor, and judged the relative height of rectangles in the lower LVF and RVF (vertical judgment task). This task dissociates retinocentric and head-centered reference frames.

Visually guided grasping. If the cortical reorganization has occurred in V1 (before the bifurcation of the visual processing stream into vision-forperception and vision-for-action subsystems) (Milner and Goodale, 1995), then vertical distortion should be evident not only in perceptual tasks, but also in a visually guided action task. The following experiment tested this prediction. B.L. sat facing a black vertical surface. He fixated a central point on the surface, and then closed his eyes while the experimenter mounted a white wooden rectangle in the lower LVF or lower RVF (centered $14^{\circ}$ horizontally from fixation, with the bottom of the rectangle aligned $12.6^{\circ}$ vertically from fixation). B.L. then opened his eyes and, while fixating the central point, reached for the rectangle. He was instructed to grasp the stimulus rectangle by the top and bottom, so that his grip aperture would reflect the height (as opposed to the width) of the stimulus. Five rectangles differing in height $\left(3.2^{\circ}, 4.8^{\circ}, 6.4^{\circ}, 8.0^{\circ}\right.$, or $\left.9.6^{\circ}\right)$ were tested; all were $3.2^{\circ}$ wide. B.L. reached with his right hand in one block of 50 trials, and with his left hand in a second block. In each block, each of the five rectangles was presented five times in the lower LVF and five times in the lower RVF, in random order.

Grip aperture (the distance between the thumb and index finger) was measured by a Polhemus (Colchester, VT) ISOTRAK II, which computed the position and orientation of sensors taped to B.L.'s thumb and index fingers as they moved through space (using low-frequency magnetic transducing technology). The sampling rate was $30 \mathrm{~Hz}$. The dependent measure was grip aperture $2 \mathrm{~cm}$ before contact with the rectangle.

\section{Retinotopic mapping}

The ventral and dorsal boundaries of V1 were mapped using a counterphase flickering $(8 \mathrm{~Hz})$ black and white checkerboard stimulus consisting of two $30^{\circ}$ wedges arranged point-to-point (see Fig. 5). The wedges stimulated either the horizontal or the vertical meridian in alternating $18 \mathrm{~s}$ blocks. Stimuli in the experimental task were solid white wedges, flickering at a rate of $8 \mathrm{~Hz}$, presented for $5 \mathrm{~s}$ on a black background (see Fig. 6). Each wedge extended from 5.6 to $12.8^{\circ}$ of visual angle from fixation, and subtended $30^{\circ}$ of polar angle ( 12 possible wedge positions, $0^{\circ}$ separation). B.L. and four control participants each completed six experimental runs; each wedge was presented five times per run in a pseudorandom sequence with the constraint that two adjacent wedges were separated by at least $10 \mathrm{~s}$, which should minimize any interacting activation between adjacent wedges.

MRI scanning was performed with a Philips (Best, The Netherlands) Intera 3T scanner in the F. M. Kirby Research Center for Functional Brain Imaging at the Kennedy Krieger Institute (Baltimore, MD). Anatomical images were acquired using a magnetization-prepared rapid acquisition gradient echo T1-weighted sequence that yielded images with a $1 \mathrm{~mm}$ isovoxel resolution [repetition time (TR), $8.1 \mathrm{~ms}$; echo time (TE), $3.7 \mathrm{~ms}$; flip angle, $8^{\circ}$; time between inversions, $3 \mathrm{~s}$; inversion time, 748 ms]. Whole-brain echoplanar functional images (EPIs) were acquired with a SENSE (MRI Devices, Waukesha, WI) head coil in 35 transverse slices (TR, $2000 \mathrm{~ms}$; run duration: 144 TRs for meridian mapping, 156 TRs for experimental task; TE, $30 \mathrm{~ms}$; flip angle, $70^{\circ}$; matrix, $64 \times 64$; field of view, $192 \mathrm{~mm}$; slice thickness, $3 \mathrm{~mm}$, no gap; SENSE factor, 2).

Brain Voyager QX software (release 1.26; Brain Innovation, Maastricht, The Netherlands) was used for the fMRI analyses. Each functional run was independently coregistered to the anatomical volume to ensure accurate functional-anatomical alignment and the in-plane data were resampled at $3 \mathrm{~mm}^{3}$ during the conversion to three-dimensional volume space. The data were then slice-time corrected and temporally filtered (high pass, 3 cycles/run; low-pass Gaussian smoothing, $2.8 \mathrm{~s}$ ). The cortical surface reconstructions in Figures 5-7 were generated using Brain Voyager's region growing and segmentation tools; the accuracy of the segmented volume was checked slice by slice in the region of $\mathrm{V} 1$ and hand corrected as necessary.

Six regression vectors were constructed by marking the temporal onset of each wedge in the LVF with a boxcar model of each respective stimulation epoch convolved with a gamma function $(\delta=2.5 \mathrm{~s}$; tau $=1.25 \mathrm{~s})$ (Boynton et al., 1996). The six resultant regression vectors were independently cross-correlated with the blood oxygenation level-dependent (BOLD) time series in each voxel within V1. The regression vector producing the maximum correlation value determined the colors depicted in Figures 6 and 7. Data from the RVF were analyzed in an analogous manner.

\section{Results}

\section{Behavior}

If the deprived V1 region (representing the upper LVF) begins to respond to stimuli that normally activate only adjacent cortex (representing the lower LVF), then stimuli presented to the lower LVF will appear vertically elongated toward and into the blind upper LVF. Accordingly, the following predictions were tested: (1) the distortion will affect the vertical (height), but not the horizontal (width), dimension of shapes presented below the blind upper LVF, (2) the vertical elongation will extend well into the blind upper LVF, and (3) the distortion will be selective to vision. The following experiments confirmed these predictions.

\section{Vertical and horizontal judgments}

In these tasks, B.L. compared the height or width of rectangles in the lower LVF and lower RVF. The results confirmed his initial reports of perceptual distortion (e.g., a circle described as a cigar extending upward) (Fig. 1) and established that the vertical, but not the horizontal, dimension was affected. Control participants were perfectly accurate in both tasks. B.L. performed well in the horizontal judgment task, accurately judging the width of the stimuli (Fig. 2a). The PSE for the horizontal judgment task was $0.34^{\circ}$ of visual angle, which is not significantly different from 0 . In the vertical judgment task, however, B.L. systematically judged the LVF rectangles to be taller than they actually were relative to the RVF rectangles (Fig. $2 b$ ). When the LVF and RVF stimuli were equal in height, B.L. virtually always judged the left rectangle to be taller; the RVF rectangle had to be $>3^{\circ}(2 \mathrm{~cm})$ taller than the LVF rectangle for him to judge them equally tall. The PSE was $3.12^{\circ}$, which is significantly $>0$.

\section{Shape judgment}

Vertical shape distortion was also observed when B.L. judged whether a single rectangle was square, taller than a square, or wider than a square. B.L. was accurate for shapes presented in the lower RVF, but systematically overestimated the height of rectangles in the lower LVF. For example, when the rectangle was $3.2^{\circ}$ $\times 3.2^{\circ}$, B.L. always reported "taller than a square." The PSE was $1.09^{\circ}$, which is significantly different from 0 . The amount of overestimation appears somewhat smaller than in the vertical judg- 
Stimuli:
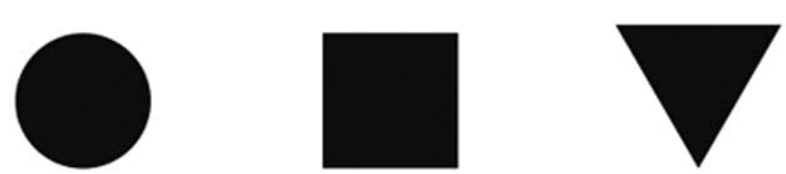

Drawings \& Descriptions:

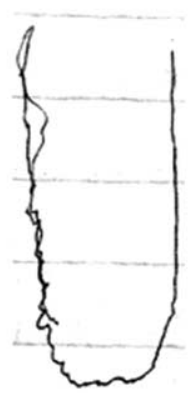

"Cigar-Like"

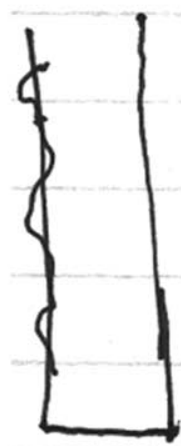

"Rectangle"

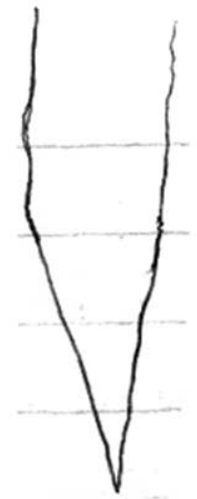

"Pencil-Like"
Figure 1. B.L.'s drawings and descriptions. For example, B.L. described a circle as a cigar shape extending toward the ceiling, a square as a tall rectangle, and a triangle with the point facing down as a pencil standing upright. B.L. described all vertically elongated shapes as "feathering out at the top."

ment experiment, perhaps because of a procedural difference between the vertical judgment and shape judgment tasks. Because the "wider than a square" shapes had to be shorter than a square in the vertical dimension, the top edge of the shapes in the shape judgment experiment were presented lower in the visual field than the top of most rectangles in the vertical judgment experiment. Given that shape distortion decreased with vertical distance from the blind upper LVF (see below, Extent of distortion), the shape judgment distortions may have been smaller than the vertical judgment distortions because the shape judgment stimuli were farther from the blind area.

\section{Extent of distortion}

In this task, a square was presented in the lower LVF or RVF, and B.L. reported the point on a numerical scale with which the top of the stimulus was aligned. The results confirmed the vertical elongation in the lower LVF, and most importantly demonstrated that lower LVF stimuli were perceived as extending well into the blind upper LVF. B.L. was accurate for lower RVF stimuli, but systematically overestimated the height of lower LVF stimuli. For example, when the top of the square appeared on the horizontal meridian in the LVF, the correct response was 8, but B.L.'s mean response was 11.3 , indicating that he perceived the square as extending $5^{\circ}$ vertically into the scotoma (Fig. 3 ). This is precisely what one would predict given that the deprived cortex representing the upper LVF has become responsive to stimuli presented to the lower LVF.

Similarly, when the top of the square was $4.8^{\circ}$ below the horizontal meridian in the LVF (i.e., the top edge aligned with the scale position 5), B.L.'s mean response was 7.6, indicating that he perceived the square as $3.2^{\circ}$ taller than it actually was. And when the top of the square was $9.6^{\circ}$ below the horizontal meridian in the LVF, the correct answer was 2, but B.L.'s mean response was 3.5 , implying a perceived vertical elongation of $1.6^{\circ}$.

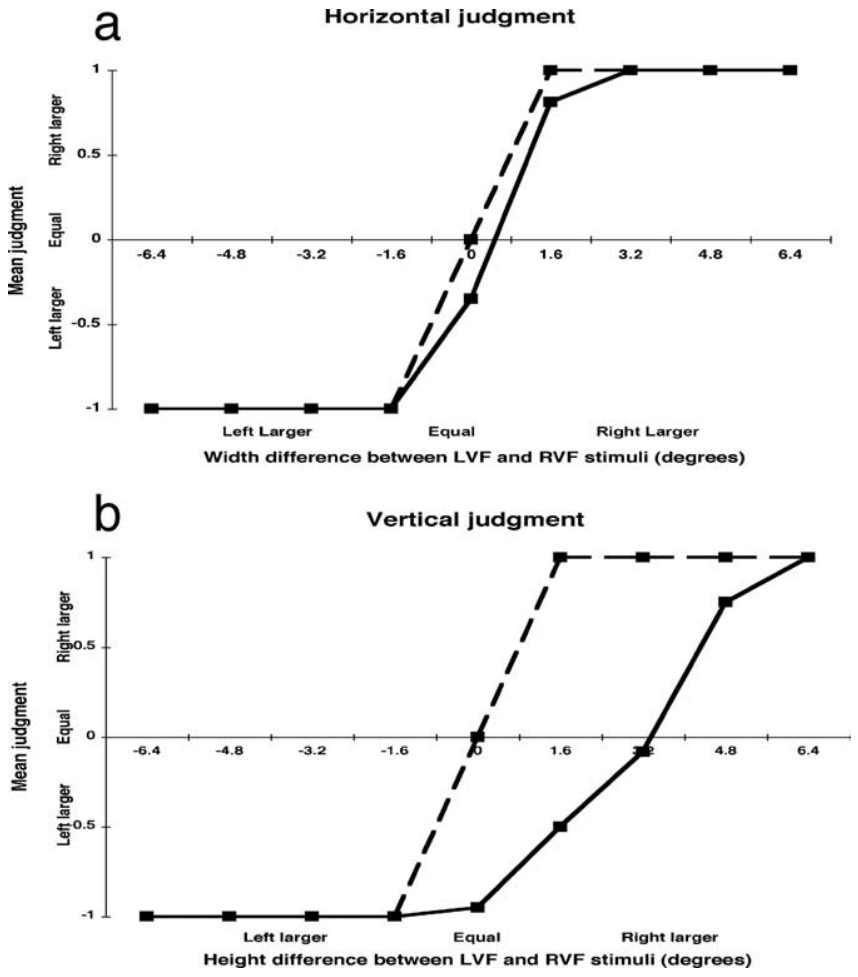

Figure 2. Visual shape perception. The dashed line depicts control participant's performance and the solid line shows B.L.'s performance. $\boldsymbol{a}$, Horizontal judgment of rectangles. $\boldsymbol{b}$, Vertical judgment of rectangles. The rightward shift of the curve for B.L. indicates that he overestimated the height of rectangles presented in the lower LVF.

The extent of vertical distortion decreased with the distance of the stimulus from B.L.'s blind upper LVF. An ANOVA revealed a significant effect of distance from the scotoma $\left(F_{(2,141)}=11.03\right.$; $p<0.001$ ), and contrasts revealed that the amount of elongation significantly decreases at each position as stimuli are moved farther from the horizontal meridian. This pattern is also consistent with cortical reorganization, given that effects of reorganization should be most pronounced for stimuli presented near the scotoma (i.e., stimuli that project to cortex immediately adjacent to the deafferented cortex).

\section{Tactile shape perception}

Results from the tactile judgment tasks revealed that B.L.'s perceptual distortion was selective to vision. Both the vertical and horizontal tactile judgments were accurate. The PSEs for these experiments were $-0.36^{\circ}$ and $-0.52^{\circ}$, neither of which is significantly different from 0 .

Overall, the results from the visual and tactile shape perception tasks confirmed the predictions following from the cortical reorganization hypothesis: B.L.'s perceptual distortion selectively affected the vertical dimension (height) of visually presented shapes, such that he perceived shapes as extending toward and into the blind upper LVF. Next, we proceeded to investigate the cortical locus of the distortion.

\section{Visual perception of spacing}

If the cortical reorganization has occurred in V1, then the distortion should affect perception not only for shape, but also for other spatial properties such as the vertical spacing between stimuli. B.L. systematically judged the spacing between lines in the lower LVF to be greater than that between lines in the lower RVF. The PSE was $1.07^{\circ}$, which is significantly different from 0 . The 


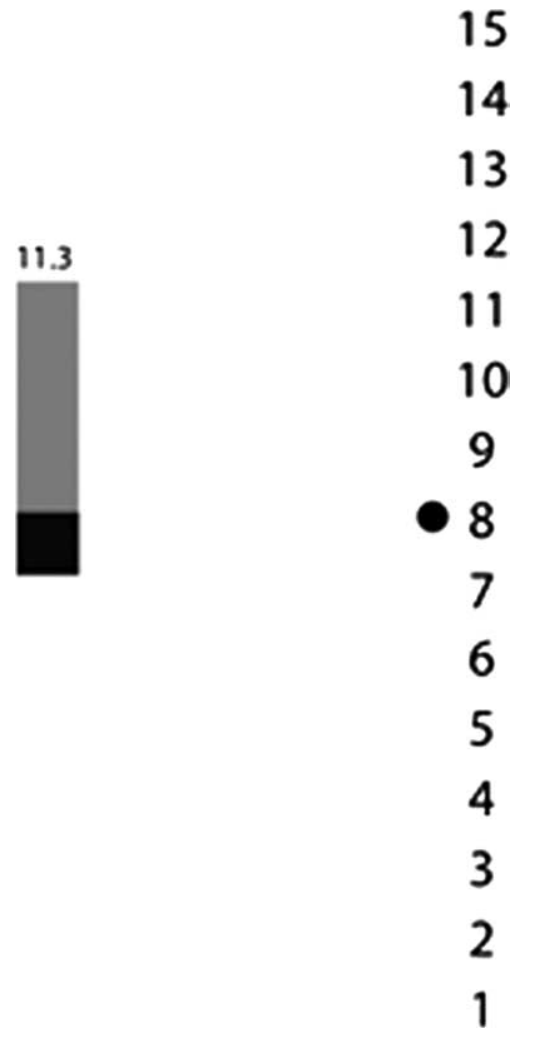

Figure 3. Example results for extent of distortion experiment. The solid black square indicates the stimulus, and the gray area denotes B.L.'s perceived vertical elongation beyond the top of the stimulus and into the scotoma.

amount of overestimation appears somewhat smaller than in the vertical judgment experiment, perhaps because of a procedural difference between the vertical judgment and spacing tasks. Because B.L. had difficulty seeing horizontal lines presented close to the scotoma, the top line of the distance stimuli was presented lower in the visual field than the top of most rectangles in the vertical judgment experiment. Given that shape distortion decreases with vertical distance from the blind upper LVF (see above, Extent of distortion), the distance distortions may have been smaller than the shape distortions because the distance stimuli were farther from the blind area. Note, however, that this amount of distortion in this task is nearly identical to that found in the shape judgment task. In both of these tasks, the stimuli were presented in the same location.

\section{Frame of reference}

Because V1 is retinotopically organized, B.L.'s perceptual distortions should reflect a retinocentric reference frame, and not a head-centered, body-centered, or environment-centered frame, if the reorganization locus is V1. Results from the frame-ofreference tasks confirmed this prediction. When B.L. observed lower LVF stimuli with his head tilted $90^{\circ}$ to the right, he described the shapes as elongated toward the right edge of the computer screen (toward and into the upper LVF). For example, a circle was described as "a cigar facing the wall." This result implies that the perceptual distortion arises in a retinocentric or headcentered frame of reference. When B.L.'s head was upright but turned to the left or right while he fixated the center of the computer monitor, he exhibited vertical distortion for lower-LVF stimuli, regardless of his head position (Fig. 4). The PSE was significantly $>0$ in both the head left condition $\left(2.08^{\circ}\right)$ and the

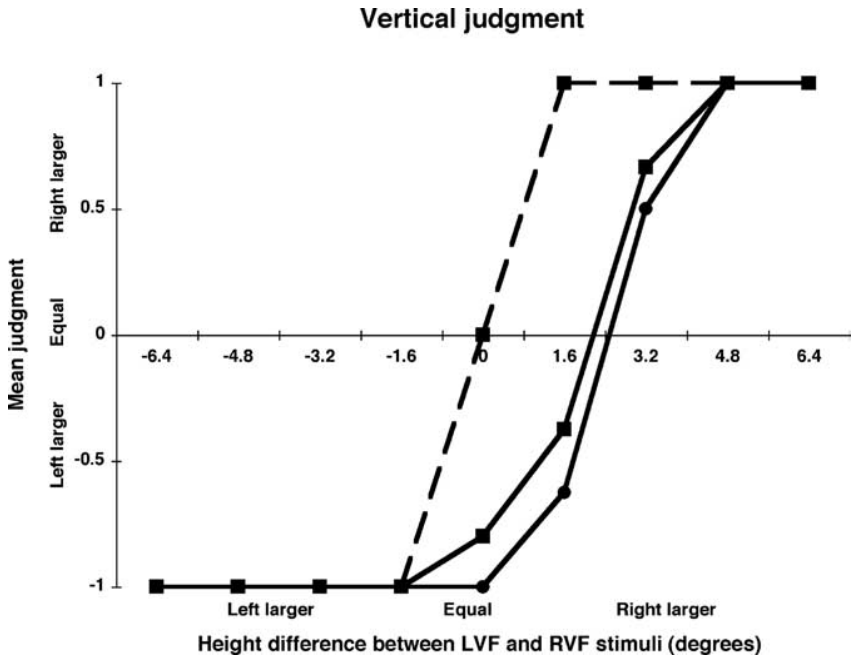

Figure 4. Frame of reference. The dashed line depicts control participant's performance. The solid line with a square shows B.L.'s performance while his head was turned left and his eyes were fixated on a central point, and the solid line with a circle depicts B.L.'s performance while his head was turned right and his eyes were fixated on a central point. The rightward shift of both curves for B.L. indicates that he overestimated the height of rectangles presented in the lower LVF.

head right condition $\left(2.73^{\circ}\right)$, and not different from one another. These findings establish that the distortion arose in a retinocentric and not a head-centered reference frame.

\section{Visually guided grasping}

Milner and Goodale (1995) proposed that, subsequent to early cortical visual areas, the visual system bifurcates into "vision for perception" and "vision for action" subsystems. If cortical reorganization has occurred in V1, before the bifurcation, then vertical distortion should be evident not only in perceptual tasks, but also in visually guided action tasks, such as reaching for or grasping an object. While reaching toward an object with the aim of grasping it, normal individuals scale their grip aperture (the distance between thumb and index finger) to the size of the target object, such that grip aperture increases with the target size (Jeannerod, 1986, 1988). This scaling of grip aperture is widely viewed as a prototypical function of the vision-for-action subsystem (Jakobson and Goodale, 1991; Goodale et al., 1994).

Whether B.L. was reaching with his left or right hand, his vertical grip apertures were significantly larger for stimuli in the lower LVF than for stimuli in the lower RVF $\left(t_{(99)}=7.64 ; p<\right.$ 0.001 ) (supplemental videos 1, 2, available at www.jneurosci.org as supplemental material). The average difference in grip apertures for the LVF and RVF was 1.5 centimeters $\left(\sim 2.5^{\circ}\right)$, consistent with the amount of elongation exhibited in the previously discussed perceptual tasks. This finding implies that B.L.'s distortions in representing lower-LVF stimuli arise at a level of the cortical visual system before the split into vision-for-perception and vision-for-action subsystems.

This finding addresses a possible alternative account of these results. B.L.'s stroke affected his right fusiform gyrus in addition to the optic radiations; it could be argued that such damage might play a role in the observed perceptual deficits. However, this explanation cannot account for B.L.'s deficit in visually guided grasping. Damage to the fusiform gyrus and related areas (e.g., V4), which are part of the ventral object recognition pathway and not part of the dorsal vision-for-action pathway, should affect 
only object recognition and not visually guided action (i.e., grasping) (Milner and Goodale, 1995).

Together, the behavioral results indicate that loss of input to a region of V1 leads to cortical reorganization of the deprived region. In particular, these results suggest that B.L.'s deprived region of cortex now responds to stimuli that normally activates adjacent cortex, because stimuli presented to retinal locations mapped to adjacent cortex appear to him perceptually elongated in the direction of the retinal location corresponding to the deprived cortex. Next, we set out to find neural evidence that deprived V1 has begun responding to stimuli that normally activates adjacent cortex.

\section{Retinotopic mapping}

B.L. and four control participants (all adults with normal vision) were tested with the same retinotopic mapping protocol. The ventral and dorsal boundaries of V1 were functionally defined with a standard meridian mapping procedure (Fig. 5). Defining the ventral boundary of V1 in the affected hemisphere for B.L. was possible because of some spared vision along the upper vertical meridian in the LVF (supplemental Fig. 2, available at www.jneurosci.org as supplemental material). During the experimental task, B.L. (and the control participants) maintained central fixation while passively viewing a random sequence of flickering wedges presented in one of 12 locations (Fig. 6a). Eye-tracking was performed during the scanning session; all participants maintained accurate fixation (supplemental Fig. 3, available at www.jneurosci.org as supplemental material).

For all control participants, a wedge in location 1R (upper RVF) (for labels, see Fig. $6 a$ ) activated the ventral boundary of left hemisphere V1, and a wedge in location 6R (lower RVF) activated the dorsal left hemisphere V1 boundary (Fig. 6c). Wedges in locations $2 \mathrm{R}-5 \mathrm{R}$ activated adjacent patches of cortex extending from the ventral to the dorsal aspects of left hemisphere V1, respectively. Similarly, wedges in locations $1 \mathrm{~L}-6 \mathrm{~L}$ activated the corresponding patches of right hemisphere V1 in all control participants (Fig. $6 c$ ). These patterns of activation are consistent with the known topography of V1 (Engel et al., 1994; Sereno et al., 1995).

Like the responses to wedges in locations $1 \mathrm{R}-6 \mathrm{R}$ in the control participants, the responses to these same wedges in B.L. activated the corresponding patches of left hemisphere V1 (the unaffected hemisphere) (Fig. 6b). In contrast, the pattern of activation in B.L.'s affected right hemisphere was quite different (Fig. 6b). As expected, little activation was observed in response to wedges presented in the (mostly blind) upper LVF (locations $1 \mathrm{~L}-4 \mathrm{~L}$ ); wedges presented in location 1L (near the upper vertical meridian, where B.L. has some spared vision) did activate a region corresponding to the ventral boundary of right hemisphere V1 at very low statistical thresholds (data not shown). Wedges presented in location $6 \mathrm{~L}$ activated the dorsal boundary of right hemisphere V1, as in the control participants. However, wedges in location 5L (lower LVF) strongly activated a wide swath of right hemisphere V1. This swath extended to the ventral V1 boundary, encompassing cortex that would normally receive input from the upper LVF (for split-half reliability, see supplemental Fig. 4; for
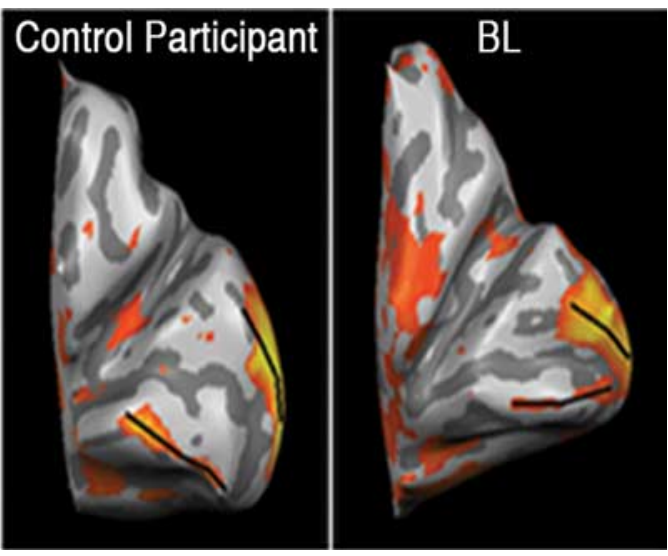

$B L$

renderings showing that the activation was not simply "jumping" from the upper to the lower region of V1 because of partial voluming or misalignment, see supplemental Fig. 5, available at www.jneurosci.org as supplemental material). Thus, a large area of ventral V1 that would normally responds to the upper LVF was not activated by upper-LVF stimuli, but was instead activated by stimuli in the lower LVF. This pattern of activation was consistent over various thresholds (supplemental Fig. $6 a$, available at www.jneurosci.org as supplemental material), ensuring that these results are not thresholding artifacts.

To quantify the extended activation to wedge $5 \mathrm{~L}$ in B.L., we computed the proportion of the cortical surface of V1 that was activated by this wedge (and 5R for comparison). Specifically, for each hemisphere of each subject, cortical surfaces were read into a custom (Qiu et al., 2006) MatLab implementation of the Dijkstra algorithm for computing distances on a convoluted surface (Dijkstra, 1959). We defined pairs of vertices such that each pair contained a vertex along the dorsal boundary of V1 and a corresponding point along the ventral boundary. Using the Dijkstra algorithm, we computed the shortest distance along the cortical surface between the defined vertices in each pair. This measurement corresponds to the cortical distance between the lower and upper bank of the V1. To compute the mean cortical extent of activation for a given wedge, we defined pairs of vertices on the dorsal and ventral edges of the region activated by the wedge. The Dijkstra computed distance between these vertices corresponds to the cortical extent of the wedge activation. For measurements of the cortical extent of both V1 and a given wedge, we chose five pairs of vertices and calculated the average distance. From these measurements, we then computed the proportion of the cortical surface of V1 that was activated by any given wedge. For normal controls, this proportions for wedge 5R [lower RVF/left hemisphere $(\mathrm{LH})]$ and 5L [lower LVF/right hemisphere $(\mathrm{RH})]$ were 0.22-0.36 (mean, 0.28; SD, 0.06), and 0.19-0.37 (mean, 0.26; $\mathrm{SD}, 0.08$ ), respectively. For B.L., wedge 5R (lower RVF/unaffected $\mathrm{LH}$ ) extended across 0.36 of the cortical distance of V1, within the normal range. However, wedge 5L (lower LVF/affected $\mathrm{RH}$ ) activated 0.56 of the cortical region designated as V1, well outside the normal range ( $\sim 4$ SDs away from the normal mean). Although these results correspond only to angular extent of the activation of a given wedge (rather than area, which in- 


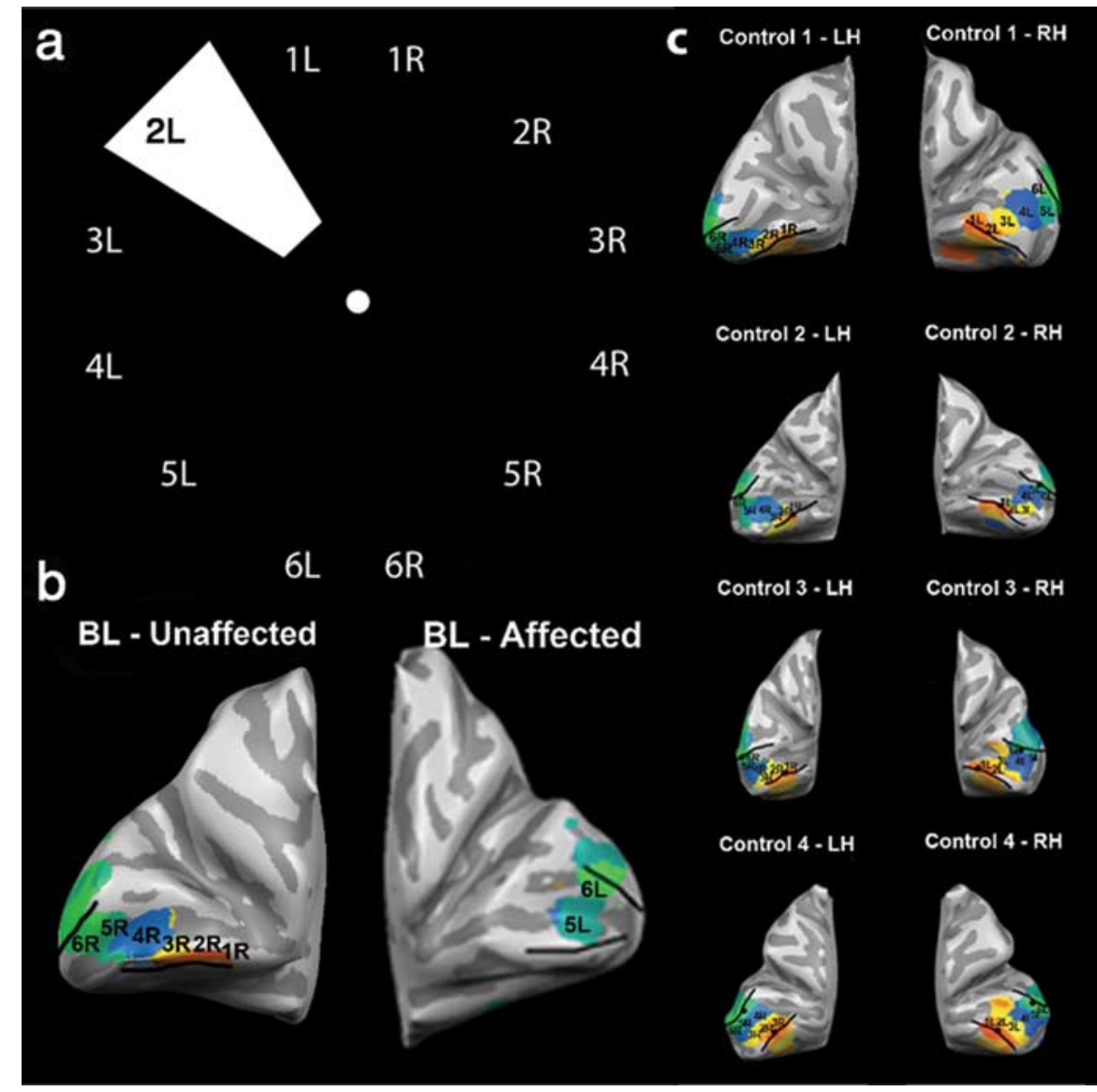

Figure 6. fMRI experimental display and results. $\boldsymbol{a}$, An example of a wedge stimulus and the tested locations during fMR scanning; the numbers referring to each wedge position were not present during scanning. $\boldsymbol{b}$, Inflated cortical surface of B.L.'s left hemisphere (unaffected) and right hemisphere (affected) occipital cortices. c, Inflated cortical surface of each control participant's left and right hemisphere occipital cortices, labeled accordingly. The upper and lower boundaries of V1, as defined by the meridian mapping procedure, are marked by solid black lines. The colors reflect the regions within V1 that were maximally correlated with each of the six stimulated locations in the RVF (which activate the left hemisphere) and the six stimulated locations in the LVF (which activate the right hemisphere). All maps shown at a threshold of $r=0.25$.

cludes angular and radial extent), our claims of cortical reorganization in B.L. predict only angular distortion, and this is precisely what we find.

Because the V1 activations shown in Figure $6 b$ show the magnitude of the maximum correlation with a wedge presented in a specific spatial location, it is possible that the area maximally stimulated by a wedge presented in location $5 \mathrm{~L}$ was also consistently activated, but to a somewhat lesser degree, by wedges presented in locations $2 \mathrm{~L}$ and $3 \mathrm{~L}$; if so, this would not be evident in the figure. In other words, we wanted to ensure that stimuli presented in location 5L (lower LVF) drove neural activity in the ventral region of V1 for B.L., whereas stimuli presented in locations $2 \mathrm{~L}$ and $3 \mathrm{~L}$ (upper LVF) failed to activate this same region (as they do in an intact visual system).

Two additional analyses were performed to check this possibility. First, we asked whether wedges $2 \mathrm{~L}$ and/or $3 \mathrm{~L}$ produced activations at various correlation thresholds (i.e., 0.3, 0.25, and 0.2 ) and found none (supplemental Fig. 6b, available at www.jneurosci.org as supplemental material). Second, we chose two regions of interest (ROIs): one at the dorsal boundary of V1 and the other at the ventral boundary of V1 (the locations were located approximately halfway in between the posterior and anterior boundaries of the V1 activation) (Fig. 7). For each ROI, we then plotted the event-related average BOLD responses time locked to the onset of wedges presented in locations $2 \mathrm{~L}, 3 \mathrm{~L}$, and $5 \mathrm{~L}$. To estimate the magnitude of the event-related BOLD response within the dorsal and ventral V1 ROIs defined for each participant, we first normalized the time series from each run by converting the data to a measure of percentage signal change by dividing the signal magnitude at each time point by the mean value of that run, subtracting 1 , and then multiplying by 100 . The event-related BOLD responses to each event type were then averaged within a temporal window extending from 0 to $16 \mathrm{~s}$ after event onset. Using this approach, the " $0 \%$ signal chance" point on the $y$-axis of the eventrelated average plots represents the mean signal across all runs.

The pattern of responses in the control brains was as expected: in the dorsal ROI, wedge $5 \mathrm{~L}$ produced the greatest response, whereas wedges $2 \mathrm{~L}$ and $3 \mathrm{~L}$ elicited little to no response. In ventral $\mathrm{V} 1$, wedge $2 \mathrm{~L}$ evoked the strongest response, whereas wedges $3 \mathrm{~L}$ and $5 \mathrm{~L}$ produced little to no response. In contrast, the pattern of responses in B.L.'s affected hemisphere was similar in the two ROIs; that is, little to no response to wedges $2 \mathrm{~L}$ and $3 \mathrm{~L}$ and a strong response to wedge $5 \mathrm{~L}$. These results confirmed that stimuli presented in location 5L (lower LVF) drove neural activity in the ventral region of V1 for B.L., whereas stimuli presented in locations 2L and 3L (upper LVF) failed to activate this same region (as they do in an intact visual system).

A final analysis examined the activation in each control participant for wedges presented in locations $1 \mathrm{~L}, 5 \mathrm{~L}$, and $6 \mathrm{~L}$. It is possible that responses to a wedge presented in location $5 \mathrm{~L}$ (lower LVF) extends broadly in the controls as well as in B.L., but that this pattern was masked by the stronger responses to wedges $2 \mathrm{~L}$, $3 \mathrm{~L}$, and/or $4 \mathrm{~L}$. However, this is not the case (supplemental Fig. 7, available at www.jneurosci.org as supplemental material). For all control participants, the activity for wedges $1 \mathrm{~L}$ and $6 \mathrm{~L}$ looked very similar to that of B.L. However, the activity for wedge $5 \mathrm{~L}$ never extended to the ventral bank of V1, as it does in B.L.

After coming out of the scanner, B.L. was asked to describe what he had seen, and these reports were consistent with the fMRI results. First, B.L. reported seeing wedges presented at three LVF locations (i.e., sometimes seeing one in the most extreme upper LVF, and two in the lower LVF), consistent with the cortical activity corresponding to wedges $1 \mathrm{~L}, 5 \mathrm{~L}$, and $6 \mathrm{~L}$, and the lack of activity in response to wedges presented in locations $2 \mathrm{~L}-4 \mathrm{~L}$. Second, he reported that wedges presented around the center of the lower LVF (just below his scotoma) extended vertically into the upper LVF such that they were larger than any RVF wedges, consistent with the swath of V1 activation corresponding to a wedge presented in location 5L. Third, he described wedges in the extreme lower LVF (location 6L) as approximately the same size as wedges presented in the RVF, and correspondingly we saw only the extent of activation normally expected for a wedge in this location (i.e., dorsal boundary of V1). And fourth, B.L. reported 
Dorsal barder

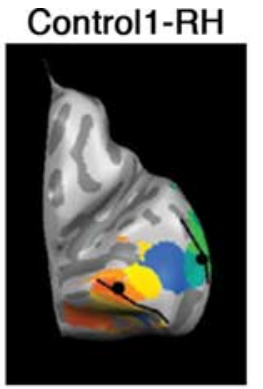

BL - Affected Hemisphere

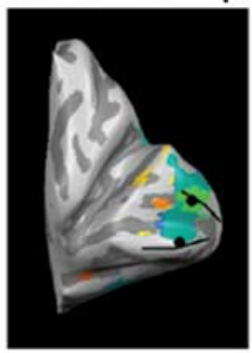

Dorsal border
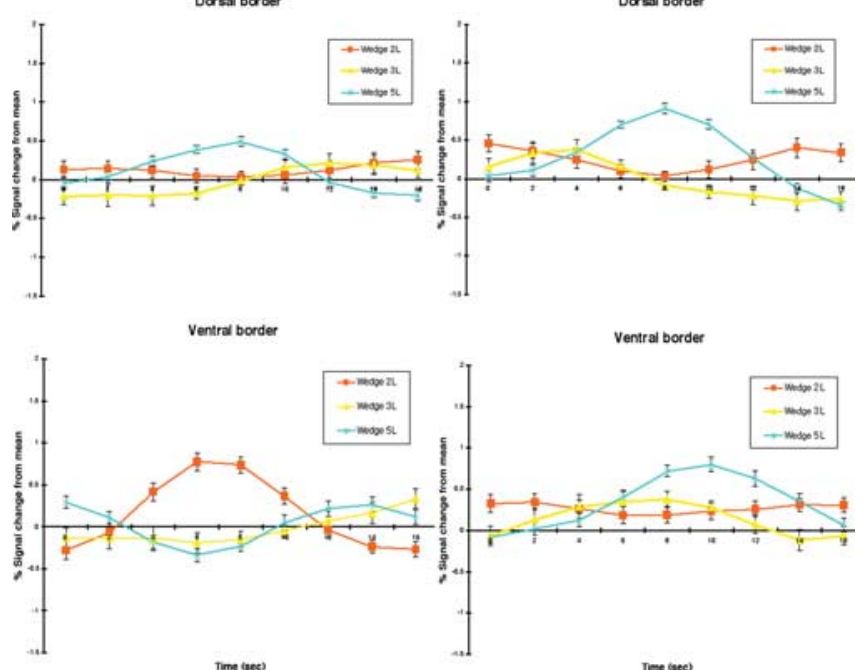

Figure 7. Event-related BOLD response time locked to the onset of wedges $2 \mathrm{~L}, 3 \mathrm{~L}$, and $5 \mathrm{~L}$. The activated voxels at a correlation threshold of 0.20 are shown for an example control (left) and B.L. (right), along with the boundaries of V1. The large dots on each boundary indicate the chosen ROls. Below each image is shown two panels, one for each of the ROls (i.e., dorsal and ventral borders). The left panels reflect the average percentage signal change across all control participants, whereas the right panel reflects that of B.L..

seeing wedges of approximately equal size at six RVF locations, consistent with the extent of activation normally expected for wedges in these locations (1R-6R).

Furthermore, the specific behavioral findings reported previously corroborate the fMRI results. First, B.L. reported stimuli as vertically elongating from the lower LVF (within the general vicinity of a wedge presented in location $5 \mathrm{~L}$ ) into the upper LVF, consistent with the spread of activation from around the dorsal boundary of V1 (cortex representing location 5L) to the ventral boundary of $\mathrm{V} 1$ (cortex normally responsive to stimuli presented in the upper LVF). Second, in the extreme lower LVF (far away from the blind area, and within the vicinity of wedge 6L), B.L. reported little to no vertical distortion, consistent with the normal activation expected for a wedge presented in location $6 \mathrm{~L}$. And third, B.L. was veridical in judging shapes presented in the RVF, consistent with the "normal" activation within the LH V1.

Although our results strongly suggest cortical reorganization, two alternative accounts should be mentioned. First, although the eye-tracking data rule out the possibility that large and systematic eye movements influenced the fMRI activations, it is possible that eye movements could partly account for B.L.'s fMRI data. For example, perhaps B.L. fixated below wedge 5L when it appeared, placing it in the upper LVF, or perhaps B.L. fixated the wedge itself to see it better. However, placing the wedge inside the scotoma cannot activate cortex because the input to V1 has been severed there. In general, alternatives based on eye movements fail to account for our observations simply because any place- ment of the stimuli that evoke activation in the deprived part of cortex imply some sort of cortical reorganization: if the deprived region is activated, it must have received input from adjacent cortex.

Second, perhaps the activation we observed in the deafferented region of V1 results from "spared islands" of V1 (or even spared projections to V1), rather than cortical organization. This argument is often used to explain the phenomenon of "blindsight," in which patients with damaged V1 have some ability to localize stimuli presented in a blind region of the visual field (Weiskrantz, 1990). However, this account bears no relevance to our results. First, our interpretation itself assumes that the upper LVF region of $\mathrm{V} 1$ is spared and that the observed activation in this region is caused by lower LVF stimuli. Second, our results cannot be explained by reference to sparing of some projections to the (deafferented) upper LVF area of V1. The crucial result we report is not that the deafferented upper LVF area of V1 is activated by upper LVF stimuli (which might suggest incomplete deafferentation); the deafferented region did not show activity in response to upper LVF stimuli. Instead, the important finding was that lower LVF stimuli activated the deafferented upper LVF area of V1. An argument involving spared projections to the upper LVF region of V1 simply has nothing to say about this finding (even if we leave aside the fact that this assumption is unsupported).

\section{Discussion}

We have presented converging behavioral and fMRI evidence showing that loss of visual input to V1 in a stroke patient (B.L.) leads to large-scale cortical reorganization. B.L. is blind in the upper LVF, and exhibits perceptual distortion in the lower LVF, stimuli appear vertically elongated, toward and into the blind region.

We hypothesized that the perceptual distortion was a consequence of cortical reorganization. If deprived cortex becomes responsive to inputs from the lower LVF such that stimuli presented in the lower LVF activated not only the V1 region representing this area, but also the adjacent region that previously received input from the upper LVF, then stimuli in the lower LVF will appear vertically elongated toward and into the blind upper LVF. Consistent with our hypothesis, behavioral testing confirmed that the distortion selectively affects the vertical dimension (height) of visually presented shapes, such that B.L. perceives shapes as extending toward and into the blind area. Additional behavioral testing established that the distortion originates early in the visual system (vertical distance as well as shape judgments are affected), the vertical distortion arises in a retinocentric frame of reference, and the deficit affects not only vision for perception, but also vision for action. Finally, fMRI studies confirmed that V1 reorganization has occurred.

Our results demonstrate that cortical reorganization can occur in the human adult visual system after loss of cortical input. This finding corroborates the numerous animal studies suggesting that V1 retains a remarkable degree of plasticity even in adulthood (Kaas et al., 1990; Heinen and Skavenski, 1991; Gilbert and Wiesel, 1992; Chino et al., 1992; Darian-Smith and Gilbert, 1995; Schmid et al., 1996; Calford et al., 2000), and contradicts previous reports of no cortical reorganization in the adult animal visual system (Horton and Hocking, 1998; Smirnakis et al., 2005a). Additionally, our results support the finding of large-scale reorganization after deafferentation of human adult visual cortex in patients with macular degeneration (Baker et al., 2005).

Our findings also provide the first clear demonstration of a direct link between cortical reorganization in the adult human 
visual system and subjective visual experience. The visual distortions described in this article may be similar to the perceptual distortions in the adult human somatosensory system (Ramachandran et al., 1992; Halligan et al., 1993; Aglioti et al., 1994). For example, Ramachandran et al. (1992) studied localization of touch sensations in human amputees, and found that stimuli applied to the face were often systematically mislocalized to the phantom arm. Ramachandran et al. (1992) attributed these "referred" sensations to cortical reorganization, arguing that when the arm area of somatosensory cortex is deafferented as a result of amputation, neurons in this area begin responding to stimuli that normally activate the adjacent face area. As a consequence, face stimulation is perceived not only as stimulation of the face, but also as stimulation of the phantom arm. Moreover, the visual distortions reported in this study may be similar to those reported by Kapadia et al. (1994) using an artificial scotoma paradigm. Our results are also consistent with those reported by Burke (1999), who suffered from a scotoma produced by a macular hole and found that a line positioned near the scotoma was perceptually deflected toward the scotoma (although retinal distortion around the macular hole could perhaps account for this result).

Other case studies on patients with damage ranging from retina to cortex describe distortions around a scotoma, but these studies are anecdotal and make no suggestion that the distortions arise from cortical reorganization (Bender and Teuber, 1947, 1948; Craik, 1966). Similar distortions have been observed in patients with metamorphopsia (also known as dysmetropsia). Although little is known about the anatomical correlates of metamorphopsia, it has been associated with a variety of structural lesions from the retina to the cerebral cortex (Young et al., 1989). Conceivably, many of the reported perceptual distortions are results of cortical reorganization. The plasticity and consequent visual distortion exhibited by B.L. may not be unusual, and would be expected to generalize to other patients with deafferented visual cortex.

How does the cortical change arise? One possible source of cortical reorganization, and the one favored by many researchers, involves the disinhibition of pre-existing long-range horizontal connections formed by the axons of pyramidal cells in V1 (Darian-Smith and Gilbert, 1994; Das and Gilbert, 1995). The idea that these horizontal connections might be responsible for the reorganization seen in the adult cat and monkey is supported by the fact that the extent of reorganization, $\sim 6-7 \mathrm{~mm}$ in diameter, approximates the length of a pyramidal cell's axon. To explain the extent of vertical elongation observed in B.L., however, horizontal connections would have to spread activation farther than the length of typical horizontal connections in primate V1 (i.e., 6-7 mm). Conceivably, a multiple-synapse chain of horizontal connections might be implicated.

A second potential mechanism for cortical reorganization involves the growth of new horizontal connections (Darian-Smith and Gilbert, 1994) rather than the unmasking of existing connections. At this time, however, our data cannot distinguish between these two alternatives. The horizontal connection account has also been proposed as a mechanism for "filling in" of real scotomas (that is, scotomas of retinal and cortical origin), artificial scotomas, the blind spot, and visual phantoms (Ramachandran and Gregory, 1991; Fiorani et al., 1992; Ramachandran, 1992; Polat and Sagi, 1994; DeWeerd et al., 1995; Kamitani and Shimojo, 1999; Zur and Ullman, 2003; Sasaki and Watanabe, 2004; Meng et al., 2005).

Finally, a third possible source of cortical reorganization is intracortical feedback to V1 from higher visual areas, such as V2 and beyond. At this point, no experiments have definitively pinpointed the respective contributions of horizontal and feedback connections in cortical reorganization.

In summary, by combining both behavioral and fMRI data, we demonstrated that adult human V1 exhibits considerable plasticity. These findings are especially important given the recent controversy regarding the occurrence of cortical reorganization in the adult visual system (Calford et al., 2005; Smirnakis et al., 2005b). More generally, shedding light on cortical reorganization contributes to our understanding of the human adult brain's capacity for change. This knowledge is critically important in designing interventions for improving recovery of function in neurological patients, as well as investigating whether cortical changes similar to those reported in this study might underlie perceptual learning and other related phenomena.

\section{References}

Aglioti S, Bonazzi A, Cortese F (1994) Phantom lower limb as a perceptual marker of neural plasticity in the mature human brain. Proc R Soc Lond B Biol Sci 255:273-278.

Baker CI, Peli E, Knouf N, Kanwisher NG (2005) Reorganization of visual processing in macular degeneration. J Neurosci 25:614-618.

Bender MB, Teuber HL (1947) Spatial organization of visual perception following injury to the brain. Arch Neurol Psychiatry 58:721-739.

Bender MB, Teuber HL (1948) Spatial organization of visual perception following injury to the brain. Arch Neurol Psychiatry 59:39-62.

Borsook D, Becerra L, Fishman S, Edwards A, Jennings CL, Stojanovic M, Papinicolas L, Ramachandran VS, Gonzalez RG, Breiter H (1998) Acute plasticity in the human somatosensory cortex following amputation. NeuroReport 9:1013-1017.

Boynton GM, Engel SA, Glover GH, Heeger DJ (1996) Linear systems analysis of functional magnetic resonance imagining in human V1. J Neurosci 16:4207-4221.

Burke W (1999) Psychophysical observations concerned with a foveal lesion (macular hole). Vis Res 39:2421-2427.

Calford MB, Tweedale R (1988) Immediate and chronic changes in responses of somatosensory cortex in adult flying-fox after digit amputation. Nature 332:446-448.

Calford MB, Wang C, Taglianetti V, Waleszczyk WJ, Burke W, Dreher B (2000) Plasticity in adult cat visual cortex (area 17) following circumscribed monocular lesions of all retinal layers. J Physiol (Lond) 524:587-602.

Calford MB, Chino YM, Das A, Eysel UT, Gilbert CD, Heinen SJ, Kaas JH, Ullman S (2005) Rewiring the adult brain (commentary). Nature 438:E3.

Chino YM, Kaas JH, Smith EL, Langston AL, Cheng H (1992) Rapid reorganization of cortical maps in adult cats following restricted deafferentation in retina. Vision Res 32:789-796.

Craik KJW (1966) On the effects of looking at the sun. In: The nature of psychology (Sherwood SL, ed), pp 98-103. Cambridge, UK: Cambridge UP.

Darian-Smith C, Gilbert CD (1994) Axonal sprouting accompanies functional reorganization in adult striate cortex. Nature 36:737-740.

Darian-Smith C, Gilbert CD (1995) Topographic reorganization in the striate cortex of the adult cat and monkey is cortically mediated. J Neurosci 15:1631-1647.

Das A, Gilbert CD (1995) Receptive field expansion in adult visual cortex is linked to dynamic changes in strength of cortical connections. J Neurophysiol 74: 779-792.

DeWeerd P, Gattass R, Desimone R, Ungerleider LG (1995) Response of cells in monkey visual cortex during perceptual filling-in of an artificial scotoma. Nature 377:731-734.

Dijkstra EW (1959) A note on two problems in connexion with graphs. Numer Math 1:269-271.

Elbert T, Flor H, Birbaumer N, Knecht S, Hampson S, Larbig W, Taub E (1994) Extensive reorganization of the somatosensory cortex in adult humans after nervous system injury. NeuroReport 5:2593-2597.

Engel SA, Rumelhart DE, Wandell BA, Lee AT, Glover GH, Chichilnisky EJ, Shadlen MN (1994) fMRI of human visual cortex. Nature 369:106.

Fiorani M, Rosa MGP, Gattass R, Rocha-Miranda CE (1992) Dynamic sur- 
rounds of receptive fields in primate striate cortex: A physiological basis for perceptual completion? Proc Natl Acad Sci USA 89:8547-8551.

Flor H, Elbert T, Knecht S, Wienbruch C, Pantev C, Birbaumer N, Larbig W, Taub E (1995) Phantom-limb pain as a perceptual correlate of cortical reorganization following arm amputation. Nature 375:482-484.

Gilbert CD, Wiesel TN (1992) Receptive field dynamics in adult primary visual cortex. Nature 356:150-152.

Goodale MA, Meenan JP, Bülthoff HH, Nicolle DA, Murphy KJ, Racicot CI (1994) Separate neural pathways for the visual analysis of object shape in perception and prehension. Curr Biol 4:604-610.

Halligan PW, Marshall JC, Wade DT, Davey J, Morrison D (1993) Thumb in cheek? Sensory reorganization and perceptual plasticity after limb amputation. NeuroReport 4:233-236.

Heinen SJ, Skavenski AA (1991) Recovery of visual responses in foveal V1 neurons following bilateral foveal lesions in adult monkey. Exp Brain Res 83:670-674.

Horton JC, Hocking DR (1998) Monocular core zones and binocular border strips in primate striate cortex revealed by the contrasting effects of enucleation, eyelid suture, and retinal laser lesions on cytochrome oxidase activity. J Neurosci 18:5433-5455.

Jakobson LS, Goodale MA (1991) Factors affecting higher-order movement planning: a kinematic analysis of human prehension. Exp Brain Res 86:199-208.

Jeannerod M (1986) The formation of finger grip during prehension: a cortically mediated visuomotor pattern. Behav Brain Res 19:99-116.

Jeannerod M (1988) The neural and behavioural organization of goaldirected movements. Oxford: Oxford UP.

Kaas JH, Krubitzer LA, Chino YM, Langston AL, Polley EH, Blair N (1990) Reorganization of retinotopic cortical maps in adult mammals after lesions of the retina. Science 248:229-231.

Kalaska J, Pomeranz B (1979) Chronic paw denervation causes an agedependent appearance of novel responses from forearm in "paw cortex" of kittens and adult cats. J Neurophysiol 42:618-633.

Kamitani Y, Shimojo S (1999) Manifestation of scotomas created by transcranial magnetic stimulation of human visual cortex. Nat Neurosci 2:767-771.

Kapadia MK, Gilbert CD, Westheimer G (1994) A quantitative measure for short-term cortical plasticity in human vision. J Neurosci 14:451-457.

Meng M, Remus D, Tong F (2005) Filling-in of visual phantoms in the human brain. Nat Neurosci 8:1248-1254.

Merzenich MM, Kaas JH, Wall J, Nelson RJ, Sur M, Felleman D (1983) Topographic reorganization of somatosensory cortical areas $3 \mathrm{~b}$ and 1 in adult monkeys following restricted deafferentation. Neuroscience 8:33-55.

Merzenich MM, Nelson RJ, Stryker MP, Cynader MS, Schoppmann A, Zook
JM (1984) Somatosensory cortical map changes following digit amputation in adult monkeys. J Comp Neurol 224:591-605.

Milner AD, Goodale MA (1995) The visual brain in action. Oxford: Oxford UP.

Polat U, Sagi D (1994) Spatial interactions in human vision: from near to far via experience-dependent cascades of connections. Proc Natl Acad Sci USA 91:1206-1209.

Pons TP, Garraghty PE, Ommaya AK, Kaas JH, Taub E, Mishkin M (1991) Massive cortical reorganization after sensory deafferentation in adult macaques. Science 252:1857-1860.

Qiu A, Rosenau BJ, Greenberg AS, Barta P, Yantis S, Miller MI (2006) Estimating linear cortical magnification in human primary visual cortex via dynamic programming. NeuroImage 31:125-138.

Ramachandran VS, Gregory RL (1991) Perceptual filling-in of artificially induced scotomas in human vision. Nature 350:699-702.

Ramachandran VS, Rogers-Ramachandran D, Stewart M (1992) Perceptual correlates of massive cortical reorganization. Science 258:1159-1160.

Sasaki Y, Watanabe T (2004) The primary visual cortex fills in color. Proc Natl Acad Sci USA 101:18251-18256.

Schmid LM, Rosa MGP, Calford MB, Ambler JS (1996) Visuotopic reorganization in the primary visual cortex of adult cats following monocular and binocular retinal lesions. Cereb Cortex 6:388-405.

Sereno MI, Dale AM, Reppas JB, Kwong KK, Belliveau JW, Brady TJ, Rosen BR, Tootell RB (1995) Borders of multiple visual areas in humans revealed by functional magnetic resonance imaging. Science 268:889-893.

Smirnakis SM, Brewer AA, Schmid MC, Tolias AS, Schüz A, Augath M, Inhoffen W, Wandell BA, Logothetis NK (2005a) Lack of long-term cortical reorganization after macaque retinal lesions. Nature 435:300-307.

Smirnakis SM, Brewer AA, Schmid MC, Tolias AS, Schüz A, Augath M, Inhoffen W, Wandell BA, Logothetis NK (2005b) Rewiring the adult brain (reply). Nature 438:E3-E4.

Sunness JS, Liu T, Yantis S (2004) Retinotopic mapping of the visual cortex using functional magnetic resonance imaging in a patient with central scotomas from atrophic macular degeneration. Ophthalmology 111:1595-1598.

Weiskrantz L (1990) Blindsight: a case study and implications. Oxford: Oxford UP.

Yang TT, Gallen CC, Ramachandran VS, Cobb S, Schwartz BJ, Bloom FE (1994) Noninvasive detection of cerebral plasticity in adult human somatosensory cortex. NeuroReport 5:701-704.

Young WB, Heros DO, Ehrenberg B.L., Hedges TR (1989) Metamorphopsia and palinopsia. Association with periodic lateralized epileptiform discharges in a patient with malignant astrocytoma. Arch Neurol 46:820-822.

Zur D, Ullman S (2003) Filling-in of retinal scotomas. Vision Res 43:971982 